\title{
Expert image analysts show enhanced visual processing in change detection
}

\author{
Tim CurRan \\ University of Colorado, Boulder, Colorado \\ Laurie Gibson and James H. Horne \\ Science Applications International Corporation, Louisville, Colorado \\ BRENT Young \\ University of Colorado, Boulder, Colorado \\ AND \\ Aloise P. Bozell \\ Science Applications International Corporation, Louisville, Colorado
}

\begin{abstract}
Expertise facilitates change detection performance, but the neural underpinnings of these benefits are unknown. Expert image analysts showed larger change-related ERP effects between about 100-200 msec after stimulus onset than did novices, which correlated with both accuracy and years of analysis experience. These results demonstrate that years of visual experience can induce fundamental changes in early visual processing which are related to change detection abilities.
\end{abstract}

Change detection provides a means for understanding how the visual system builds, stores, and attends to visual representations (Rensink, 2002; Simons \& Rensink, 2005). Expertise has been shown to enhance change detection in prolonged tasks where it could influence several stages of information processing, from initial perceptual processing through response execution (Jones, Jones, Smith, \& Copley, 2003; Werner \& Thies, 2000). Considering visual processing alone, models of change detection posit important roles for early as well as higher level visual processes (Rensink, 2002). Previously identified change-related ERP effects suggest that early visual processes may contribute to change detection (Kimura, Katayama, \& Murohashi, 2005, 2006a, 2006b, 2006c). Professional overhead image analysts, with their expertise in interpreting natural scenes, present an ideal opportunity to examine ERP effects indicative of an influence of expertise on these early processes.

The present experiment was designed to incorporate key elements of the way imagery analysts carry out their real-world change detection tasks. To familiarize themselves with a site, analysts examine several images taken from different viewpoints. As new images are acquired, analysts will compare past and present appearance of the site, focusing not on incidental changes in perspective, lighting, and so on, but on changes that indicate significant activity, such as the arrival of new vehicles or the construction of new buildings.
In the present experiment, expert image analysts and novices viewed realistic overhead images of sites such as military compounds, airports, and ports. For familiarization (see Figure 1), an initial study phase allowed the subject to view two side-by-side images of a site; this was followed by a change detection task, with pairs of images presented sequentially. The first image (S1) was from the study phase, a reminder of the original scene so that performance would not be overly dependent on memory differences between experts and novices. The second image (S2) was either an originally studied image (unchanged) or an image changed by adding, deleting, or changing significant objects such as vehicles, boats, or buildings. S2 could be the same perspective as S1 or a different one, but subjects were told to ignore perspective changes and respond strictly according to whether or not S2 was changed or unchanged from the originally studied images. The task was easy enough for both experts and novices to complete with high accuracy rates. We predicted that expertise would modulate early perceptual ERP components, time-locked to S2.

\section{METHOD}

\begin{abstract}
Subjects
Eleven expert image analysts and 11 novices participated. The experts' age range was 28 to 46 years, mean age 34 ( 3 female, 8 male). Each expert had undergone training in a basic image analysis course given to military and civilian analysts and had worked as a professional image analyst for at least 3 years $(M=8$ years). The novices'
\end{abstract}

T. Curran, tim.curran@colorado.edu 
$20 \mathrm{sec}$

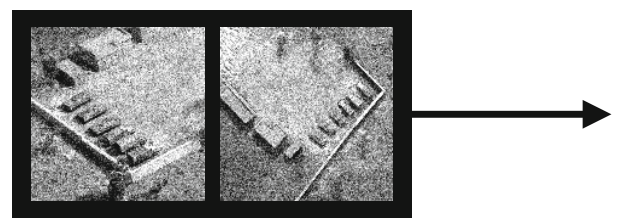

Study Phase

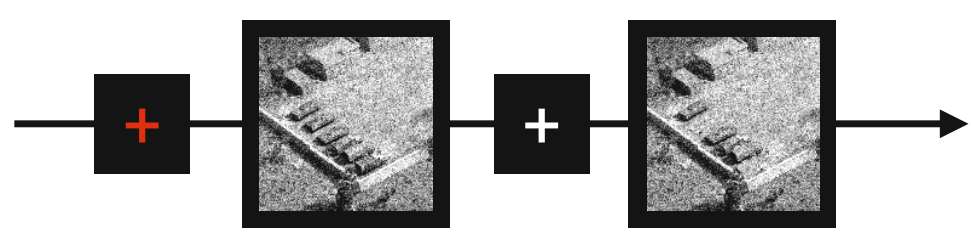

S1

S2

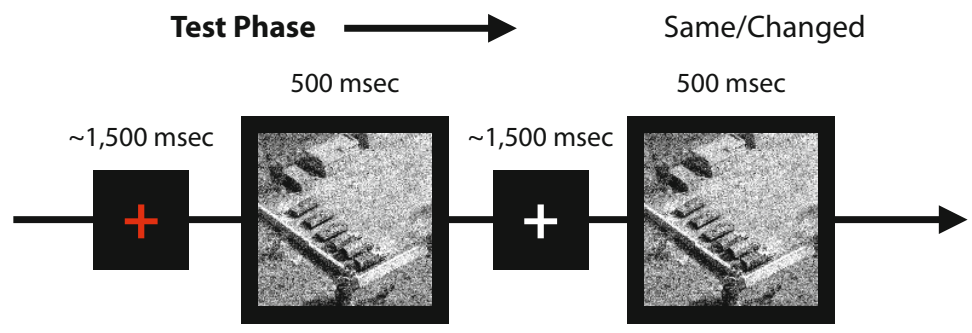

S1

S2

Same/Unchanged

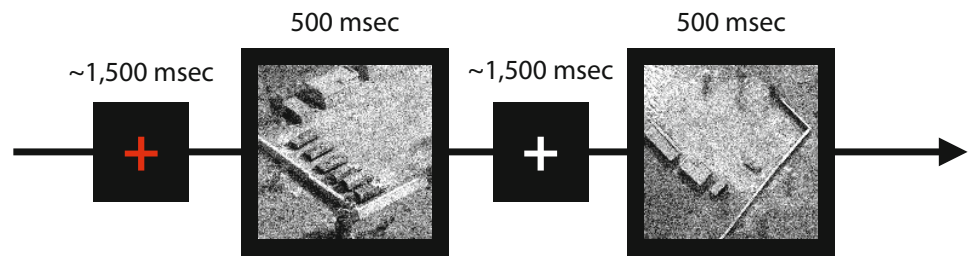

S1

S2

Different/Changed

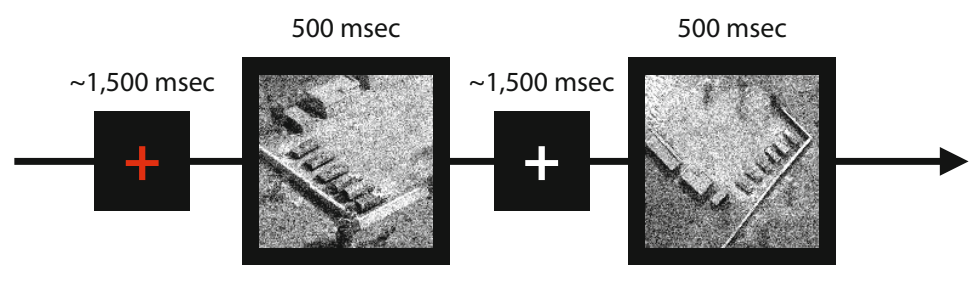

S1

S2

Different/Unchanged

Figure 1. Event timing in the study phase and first four trials of a block. Shown here are examples of (1) same perspective, changed; (2) same perspective, unchanged; (3) different perspective, changed; and (4) different perspective, unchanged trials. Scale model image provided by the National GeospatialIntelligence Agency (NGA). 
age range was 20 to 41 , mean age 35 ( 5 female, 6 male). The novices were college educated and worked in non-image-related professions. All subjects were right-handed.

\section{Materials}

Stimuli were images of sites such as military compounds, airports, and ports. Some images were individual frames from video sequences acquired from an aircraft flying around ground sites. Others were pictures taken with a digital camera of a scale model site, processed to simulate satellite images by adding Gaussian noise (e.g., Figure 1). All images were cropped to a 128-pixel square frame. Ten images from six sites were used as stimuli (60 total), and two other sites were used for practice. For each site, there were two base images taken from different viewpoints. Each site also contained four change images for each perspective. Changes included vehicles, buildings, and boats that were added, subtracted, or changed. Changes were $33 \%$ additive, $42 \%$ subtractive, and $25 \%$ mixture between addition, subtraction, and changes. The changed images were created by editing the base images using Adobe Photoshop. Because we were interested in individual differences, we chose to keep stimuli fixed to changed/unchanged conditions rather than counterbalanced or randomly assigned, so that stimulus differences would not obscure subject differences. The methods used to create the changed/ unchanged image pairs were designed to prevent systematic differences between the conditions. For additions, the original unedited image was the "changed" image. The original image was edited to remove objects by covering them with background regions taken from the same image, and the result was the "unchanged" image. The images were subsets of larger pictures, so objects were added to an image from other parts of the same picture.
\end{abstract}

\section{Procedure}

The subjects were seated in front of a computer monitor at a distance of approximately $100 \mathrm{~cm}$. An image displayed at the center of the monitor subtended a visual angle of about $2.43^{\circ}$ horizontally and $2.58^{\circ}$ vertically. The experiment consisted of six blocks. Each block had a study phase followed by a test phase with 64 trials. During the study phase, the subject viewed two base images for a site side by side on the monitor for $20 \mathrm{sec}$. Each change-detection trial began with a central red fixation cross displayed for a random interval varying from 1,250 to $1,750 \mathrm{msec}$. One of the base images from the study phase was then presented for $500 \mathrm{msec}$, followed by a white fixation cross (randomly varying from 1,250 to $1,750 \mathrm{msec}$ ), then a second image of the same site for $500 \mathrm{msec}$. The second image could be one of the two base images (the unchanged condition) or one of eight changed images of the site (the changed condition). Subjects were instructed to respond as soon as possible, and the trial terminated upon the response. If the response was slower than the 500-msec S2 duration, a central question mark appeared until the subject responded, with a 2,000-msec deadline from question mark onset. The subject pressed the "changed" key if the second image contained a significant change from the base images that were originally studied. If the second image was identical to the first, or displayed only a perspective change, the subject pressed the "unchanged" key.

In each trial, there were two independent variables: perspective and change. The second image in a pair could have the same perspective as the first or a different one, and it could show change or no change in comparison with the originally studied images. Subjects were instructed to ignore perspective differences between the first and second images of each pair, and to concentrate on meaningful changes between the second image and the originally studied images when making their change/no-change responses.

There was one block for each of the six image sets, and there were 96 trials for each of the four conditions, comprising 384 trials per session. In each block, the subject was shown 16 trials for each condition. The order of the trials within the block was random. Each subject completed two sessions, 6-14 days apart, for a grand total of 192 trials for each of the four conditions and 768 trials overall. The two sessions of the experiment were identical, with the exception that the ordering of the sets varied. A different order was used for these first and second sessions, and those orders were fixed across subjects.

\section{EEG Recording and ERP Averaging}

Scalp EEG data were collected with a 128-channel HydroCel Geodesic Sensor Net connected to an AC-coupled 128-channel, high-input impedance amplifier (200 $\mathrm{M} \Omega$, Net Amps, Electrical Geodesics Inc., Eugene, OR). Individual sensors were adjusted until impedances were less than $50 \mathrm{k} \Omega$. Amplified analog voltages (0.1- to $100-\mathrm{Hz}$ bandpass) were digitized at $250 \mathrm{~Hz}$. Recorded voltages were initially referenced to a vertex channel. EEG data were excluded from analysis if it was associated with incorrect trials or contained eye movements (electro-oculogram channel differences greater than $70 \mu \mathrm{V}$ ) or more than five bad channels (changing more than $100 \mu \mathrm{V}$ between samples, or reaching amplitudes over $200 \mu \mathrm{V}$ ). Data from individual bad channels were replaced using a spherical interpolation algorithm.

ERPs were time locked to the second image of each pair. The mean number of acceptable trials retained for ERP averaging per condition per subject was 172.5 (experts, $M=174$, range, 172-175; novices, $M=173$, range, 171-175). Voltages were rereferenced offline into an average reference, corrected for the polar average reference effect (Junghöfer, Elbert, Tucker, \& Braun, 1999). ERPs were baseline corrected for the 200-msec interval prior to the presentation of S2 and digitally low-pass filtered at $40 \mathrm{~Hz}$.

\section{RESULTS}

All dependent measures - accuracy, reaction time (RT), and ERP amplitudes - were analyzed in a group (expert, novice) $\times$ perspective (same, different $) \times$ change (changed, unchanged) ANOVA. ERP ANOVAs also included an additional factor of hemisphere (left, right).

Accuracy was higher in the same-unchanged condition $(99 \%)$ than in all others $(97 \%)$ [change $\times$ perspective interaction, $\left.F(1,20)=10.29, M S_{\mathrm{e}}=.002, p<.01\right]$. Change effects on RT were larger in the different (unchanged, $955 \mathrm{msec}$; changed, $836 \mathrm{msec}$ ) than in the same perspective (unchanged, $885 \mathrm{msec}$; changed, $833 \mathrm{msec}$ ) conditions [change $\times$ perspective interaction, $F(1,20)=32.75$, $\left.M S_{\mathrm{e}}=1,461, p<.001\right]$. No other accuracy or RT effects, including group effects or interactions, were statistically significant, although trends for experts to have higher accuracy $(98 \%$ vs. $97 \%)$ and faster RT (854 vs. $901 \mathrm{msec})$ were observed.

ERP results are shown in Figure 2. Inspection of the ERPs suggested that change effects on early perceptual components were most prominent at electrode locations O1 (left hemisphere, \#70) and O2 (right hemisphere, \#83), so analyses focused on these channels. However, topographic maps of these differences confirm that these effects were more widespread (Figures 3A-3D). These early change effects appeared to be coincident with the $\mathrm{P} 1$, N1, and P2 components. Change effects occurring later and encompassing the $\mathrm{P} 3$ component were also evident, and were analyzed at standard parietal locations P3 and P4, where the P3 component is typically observed. For each component, mean amplitude was computed within a 

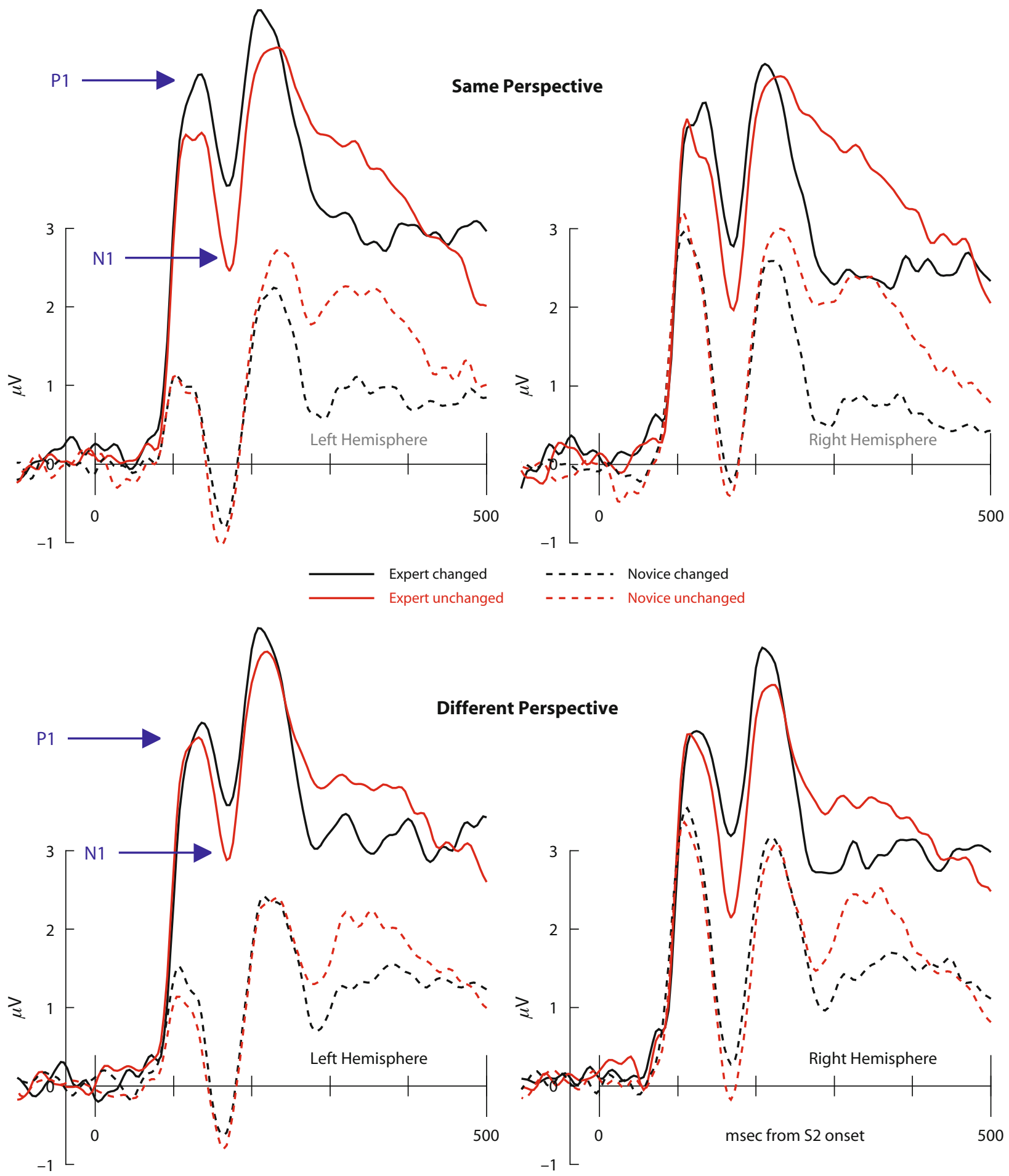

Figure 2. Grand average ERPs from channels $\mathrm{O1}$ (left) and $\mathrm{O} 2$ (right) in the same (top) and different (bottom) perspectives. ERPs are time locked to the onset of the second image (S2). 
P1 (104-140 msec) N1 (160-184 msec)

A. Expert: Same Changed - Same Unchanged

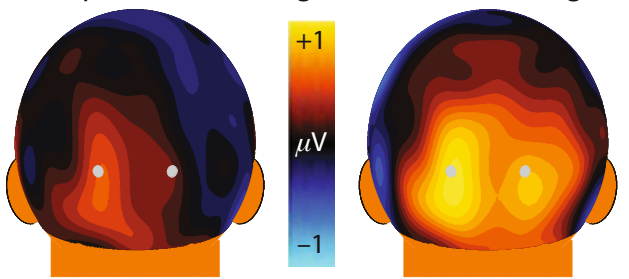

B. Novice: Same Changed - Same Unchanged

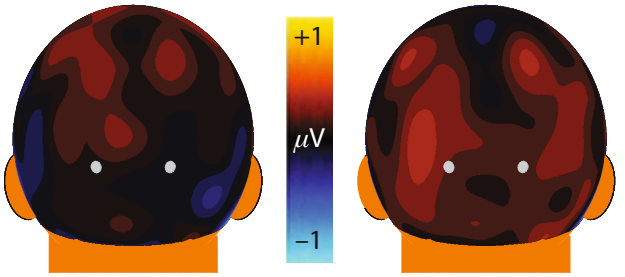

C. Expert: Diff Changed - Diff Unchanged

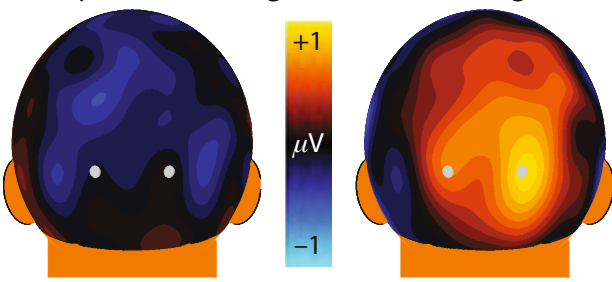

D. Novice: Diff Changed - Diff Unchanged

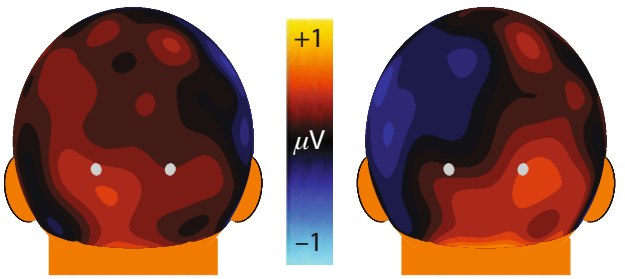

E. Expert: Same Unchanged

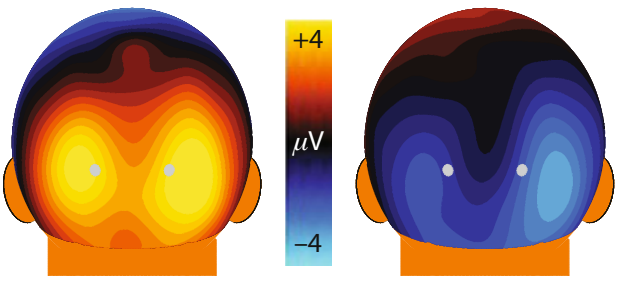

F. Novice: Same Unchanged

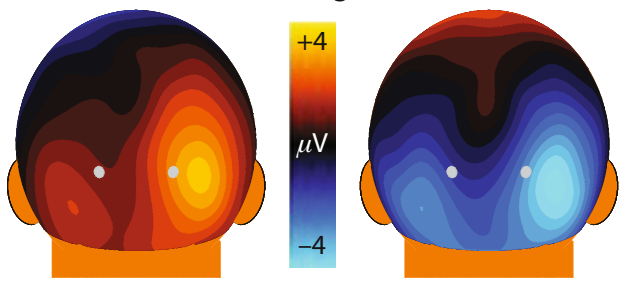

Figure 3. Topographic maps showing changed - unchanged difference ERPs (A-D) and ERPs in the unchanged/same condition (E and F) within the P1 (left) and N1 (right) windows. Channels $\mathrm{O} 1$ and $\mathrm{O} 2$ are marked with gray circles. Baseline correction was from -200 to 0 msec for all maps except the unchanged/same $\mathrm{N1}$ maps (right, $\mathrm{E}$ and $\mathrm{F}$ ), which were baseline corrected over the $P 1$ interval (104-140 msec) to remove prior P1 influences. temporal window defined as $1 S D(\mathrm{P} 1, \mathrm{~N} 1, \mathrm{P} 2)$ or $2 S D \mathrm{~s}$ (P3) around the peak latency of the component (adjusted to the nearest $250-\mathrm{Hz}$ sample). Thus, P1 amplitude was calculated between 104-140 msec, N1 amplitude was calculated between 164-184 msec, P2 amplitude was calculated between 204-256 msec, and P3 amplitude was calculated between $468-648 \mathrm{msec}$.

P1 amplitude was more positive for changed than for unchanged images $\left[F(1,20)=4.77, M S_{\mathrm{e}}=.58, p<.05\right]$ and this change effect interacted with group and perspective $\left[F(1,20)=4.46, M S_{\mathrm{e}}=.49, p<.05\right]$. Pairwise comparisons indicated that the change effect was only significant for experts when the perspective was the same $(p<$ $.05)$. The main effect of group was marginally significant $\left[F(1,20)=3.81, M S_{\mathrm{e}}=133, p=.065\right]$. N1 amplitude also was more positive for changed than for unchanged images $\left[F(1,20)=15.24, M S_{\mathrm{e}}=1.56, p<.001\right]$, and this change effect interacted with group $[F(1,20)=5.28$, $\left.M S_{\mathrm{e}}=1.56, p<.05\right]$. The change effect was significant for experts $\left[F(1,10)=13.30, M S_{\mathrm{e}}=.50, p<.01\right]$ but not for novices $\left[F(1,10)=2.33, M S_{\mathrm{e}}=1.96\right]$. The main effect of group was marginally significant $[F(1,20)=$ 4.22, $\left.M S_{\mathrm{e}}=220, p=.053\right]$. P2 amplitude showed an interaction between change, perspective, and hemisphere $\left[F(1,20)=5.05, M S_{\mathrm{e}}=.10, p<.05\right]$. The $\mathrm{P} 3$ amplitude was more positive for changed than for unchanged images $\left[F(1,20)=58.06, M S_{\mathrm{e}}=2.66, p<.0001\right]$. Because reliable group effects or interactions were observed only for the $\mathrm{P} 1$ and $\mathrm{N} 1$, these components alone are considered further.

The $\mathrm{P} 1$ and $\mathrm{N} 1$ effects reported above might reflect separate modulations of the processes primarily responsible for P1 and N1 generation, with change enhancing P1 yet reducing N1; or it could reflect a single effect of change distinct from the primary P1/N1 generators yet summing to influence the observed amplitudes (changed more positive than unchanged). To address these possibilities, we compared the topography of the P1 and N1 components with the topography of the change effects (Kimura et al., $2005,2006 b, 2006 \mathrm{c}$ ), focusing on the experts' results in the same perspective conditions that showed change effects at both times. P1 and N1 topography was assessed in the unchanged/same condition (Figure 3E), and N1 topography was computed after baseline correcting over the 104- to $140-\mathrm{msec} \mathrm{P} 1$ period to remove prior P1 influences. Change effects were assessed by taking the difference between changed and unchanged images with the same perspective in each time window (Figure 3A; P1d, N1d). A component (P1 vs. P1d) $\times$ hemisphere $X$ electrode (1-59, excluding midline channels) repeated measures ANOVA revealed a significant component $X$ electrode interaction $\left[F(58,580)=10.05, M S_{\mathrm{e}}=1.60\right.$, $p<.0001]$. This and all $p$ values were corrected with the Greenhouse-Geisser correction for inhomogeneity of covariance. This interaction remained significant when the main effect of component was removed by range normalization (McCarthy \& Wood, 1985) $[F(58,580)=4.99$, $\left.M S_{\mathrm{e}}=.229, p<.002\right]$. No significant component $\times$ electrode interactions were observed when N1 and N1d were 
compared $\left[F(58,580)=2.220, M S_{\mathrm{e}}=2.218\right.$; normalized, $F<1, M S_{\mathrm{e}}=.32$; both $\left.p \mathrm{~s}>.10\right]$. A direct comparison of the topography of the change effect at each time (P1d vs. N1d) also failed to reveal any electrode $\times$ component interactions $\left[F(58,580)=2.06, M S_{\mathrm{e}}=.60\right.$; normalized, $F(58,580)=2.00, M S_{\mathrm{e}}=.46$; both $\left.p \mathrm{~s}>.10\right]$. The observation that the topography of the change effect did not differ across time (P1d vs. N1d), and that the topography of the P1d was significantly different from the P1 topography, is consistent with the change effect not being merely an enhancement of the P1. The failure to find significant topographic differences between the N1 and N1d leaves open the possibility that the N1d is a modulation of the N1, but the most parsimonious account of our data is that we observed a single effect of change distinct from the primary $\mathrm{P} 1 / \mathrm{N} 1$ generators yet summing to influence the observed amplitudes (changed more positive than unchanged). This interpretation is consistent with previous reports of a change-related positivity (CRP) (Kimura et al., 2005, 2006a, 2006b, 2006c).

We examined the correlations of accuracy and RT with an aggregate measure of the CRP, encompassing both the P1 and N1 windows reported above. Each subject's CRP effect was summarized by a single changed-unchanged ERP difference score, averaged across all conditions from 104 to $184 \mathrm{msec}$ (the beginning of the P1 window to the end of the N1 window). The CRP was positively correlated with accuracy $(r=.44, p<.05$, across all subjects; Figure 4A), but not with RT $(r=.03)$. When experts were considered alone, years of experience significantly correlated with the CRP $(r=.65, p<.05$, Figure 4B). Correlations with age were also examined, but only the correlation with years of experience approached significance $(r=.55, p=.08)$. Critically, age did not correlate with the CRP $(r=-.03)$, so age cannot explain the significant correlation between the CRP and years of experience.

Further analyses addressed the possibility that the larger CRP for experts than for novices is an artifact of the seemingly large, but marginally significant, main effects of group on P1 and N1 amplitudes. First, the primary P1 and $\mathrm{N} 1$ change effects remained reliable when amplitudes were normalized to remove group differences. Second, Figure 4C shows that there is a trend toward the CRP increasing with mean 104- to 184-msec amplitude for experts $(r=.32, p=.35)$, but no such trend for novices $(r=-.04)$. Thus, there does not appear to be any reliable influence of overall amplitude on the CRP.

\section{DISCUSSION}

The present results suggest that expertise is associated with a striking enhancement of the brain's early response to visual change. Other studies have documented the behavioral advantages of expertise in change detection (Jones et al., 2003; Werner \& Thies, 2000), but the present research establishes that expertise affects visual processes starting around $100 \mathrm{msec}$ after a changed stimulus is presented. When engaged in a change detection task

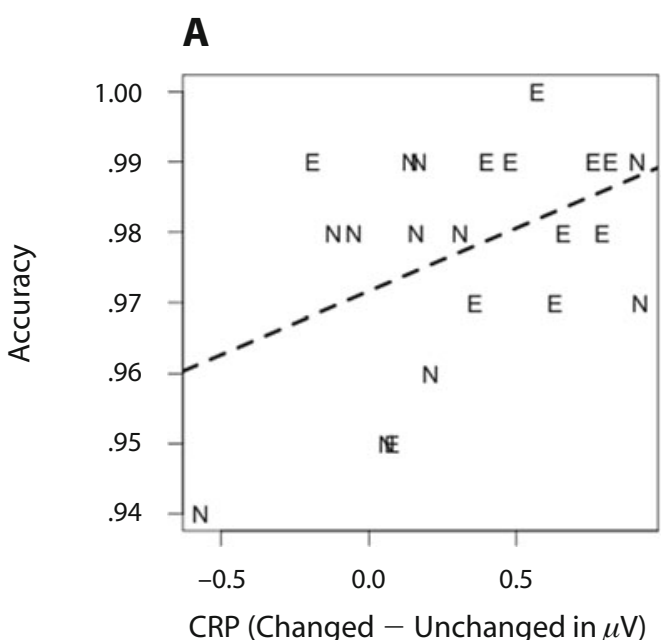

B

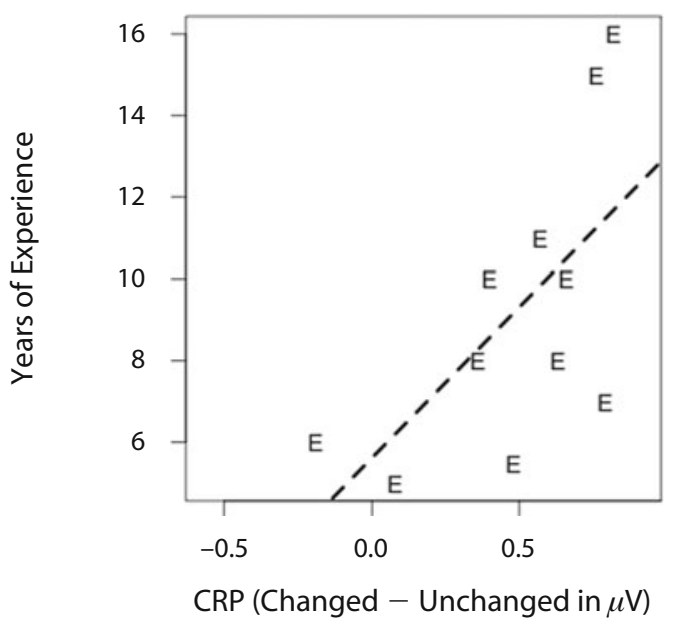

C

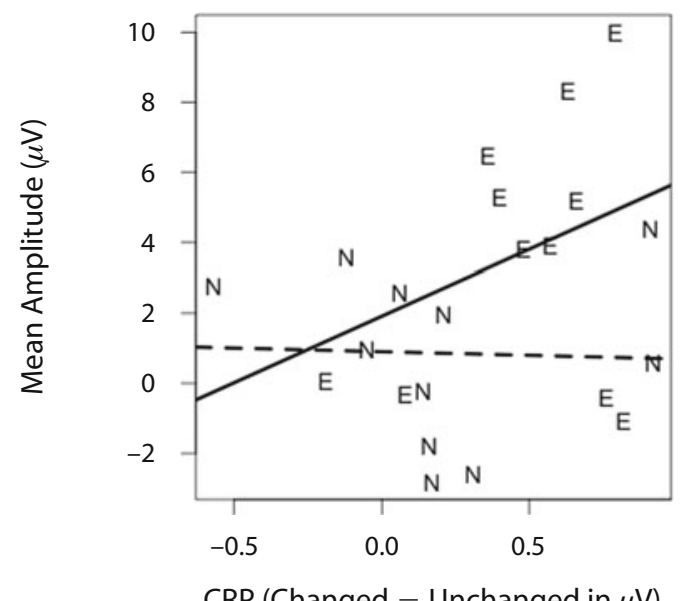

Figure 4. Scatterplots showing the significant positive correlations of the change-related positivity (CRP) with (A) accuracy across all subjects, and $(B)$ years of image analysis experience in expert subjects. (C) Scatterplot showing a trend toward the CRP increasing with mean amplitude for experts (solid line), but not for novices (dashed line). E, expert; $N$, novice. 
involving realistic overhead images, expert image analysts showed larger ERP amplitude differences between changed and unchanged stimuli about $100-180 \mathrm{msec}$ after stimulus onset than did novices, for whom these early differences were not statistically significant. Similar early change-related ERP effects have been observed in nonexpert subjects, the CRP, but these typically involve very simple stimuli (Kimura et al., 2005, 2006a, 2006b, 2006c). Stimulus complexity is one determinant of the time course of the ERP response to change (Koivisto \& Revonsuo, 2003). The present results further demonstrate that expertise can similarly modulate this time course, perhaps because the stimuli are simpler for experts. Although experts and novices showed similar overall performance, early change effects were positively correlated with accuracy across all subjects, and positively correlated with experts' years of experience. Other research has documented expertise-related changes on ERPs (Busey \& Vanderkolk, 2005; Gauthier, Curran, Curby, \& Collins, 2003; Rossion, Collins, Goffaux, \& Curran, 2007; Tanaka \& Curran, 2001), but this is the first study to specifically relate early visual effects to change detection ability and duration of related experience by demonstrating that the ERP change effects correlated with both accuracy and years of image analysis experience.

Prior research on the CRP has helped delineate its functional significance in ways that may have implications for understanding the present expertise effects. Kimura et al. $(2005,2006 \mathrm{~b}, 2006 \mathrm{c})$ have argued that the CRP reflects the activity of a memory comparison process, rather than merely an attentional modulation of the P1 and N1, because the $\mathrm{P} 1$ and $\mathrm{N} 1$ components have different topographies than the CRP does. The present results suggest that the change effect was topographically different from the $\mathrm{P} 1$, but not statistically different from the N1. The present results are most parsimoniously described as the effects of a single CRP superimposed upon the P1 and N1 results. Thus, the present expertise effects may reflect experiencedependent tuning of a visual memory process, rather than just greater attention to changes shown by experts. However, it should be noted that the CRP is typically observed in conditions where changes are incidental to task performance (Kimura et al., 2005, 2006b, 2006c) rather than within an explicit change detection task, as done here. Thus, one might expect the CRP to show incidental effects to the present perspective differences, but no such effects were observed. Again, the greater complexity of the present stimuli may be responsible for the different results since, to our knowledge, the CRP has never been observed with naturalistic images. Perhaps the experience of experts allowed the processes underlying the CRP to detect changes in more complex stimuli, as well as to focus on primarily task-related changes.

Our results could reflect components other than the CRP. First, a similar latency-change related negativityCRN, or visual mismatch negativity - is often observed (Pazo-Alvarez, Cadaveira, \& Amenedo, 2003). The present results are not consistent with the CRN, which is typically more negative for changed than for unchanged stimuli, rather than the more positive response to change observed here. Furthermore, the CRN is typically observed when changes are infrequent "oddball" events rather than equiprobable with unchanging events, as observed here (Kimura et al., 2006c). As a second alternative, the greater negativity to unchanged stimuli may reflect enhanced attention to these images, as is often reflected in posterior negativities (Folstein \& Van Petten, 2008), which may also explain the slower RT to unchanged images (e.g., rechecking for changes). Future research will need to tease apart these possibilities by examining expertise effects in the context of other manipulations known to selectively influence specific change-related ERP components.

Limitations of the present research include lack of a control condition with images outside the experts' domain and high accuracy rates. First, a control condition would allow us to examine the specificity of the expertise effects, although unreported analyses indicated that the P1 to the fixation cross did not differ between groups, so the observed differences are unlikely to reflect mere motivational differences between groups that would be expected to enhance sensory responses to all stimuli. Interpretation was also complicated by a trend toward more positive amplitudes overall for experts than for novices, similar to what has been reported in at least one other ERP study of language-related expertise (Wong, Gauthier, Woroch, DeBuse, \& Curran, 2005), where group differences extended to control stimuli (false fonts), so probably reflected greater motivation by a bilingual than monolingual group. Expertise studies are probably best designed when two matched groups of experts are compared (e.g., Tanaka \& Curran, 2001), because each group will be similarly motivated, and the expert stimuli for one group is the novice stimuli for the other and vice versa. Second, high accuracy rates did not allow us to examine change blindness and questions of visual awareness, as has been done in previous ERP studies comparing conditions with detected versus undetected changes rather than detected changes versus no changes, as in the present case (e.g., Mazza, Turatto, \& Sarlo, 2005). Furthermore, high accuracy rates limited our ability to detect group differences in performance. We intentionally made the task easy for both experts and novices, but expected that RT would significantly differ between groups. However, the correlation observed between accuracy and the CRP suggests that the presently observed ERP results are behaviorally relevant to expertise.

\section{AUTHOR NOTE}

This research was supported by the Defense Advanced Research Projects Agency under Contract HM1582-05-C-0040. We thank Casey DeBuse, Brion Woroch, Kristin Becker, Alexis Billings, Chris Bird, Jeanne Doyle, Sarah Jirkovsky, and Jessica Patcher for research assistance; Amy Kruse, Joshua Schulman, and Jeffrey Kretsch for their suggestions on various stages of the research; and Marlene Behrmann, Erika Nyhus, Lisa Scott, Dan Simons, Jim Tanaka, and Katharine Tepe for comments on a draft of this article. Scale model images were produced by SAIC for the National Geospatial-Intelligence Agency under Contract DASG6002-D-0006. This document was approved by DARPA for Public Release, Distribution Unlimited. Correspondence concerning this article should be addressed to T. Curran, Department of Psychology and Center for Neuro- 
science, University of Colorado, Boulder, CO 80309-0345 (e-mail: tim .curran@colorado.edu).

\section{REFERENCES}

Busey, T. A., \& VANDERKolK, J. R. (2005). Behavioral and electrophysiological evidence for configural processing in fingerprint experts. Vision Research, 45, 431-448.

Folstein, J. R., \& VAn Petten, C. (2008). Influence of cognitive control and mismatch on the N2 component of the ERP: A review. Psychophysiology, 45, 152-170.

Gauthier, I., Curran, T., Curby, K. M., \& Collins, D. (2003). Perceptual interference supports a non-modular account of face processing. Nature Neuroscience, 6, 428-432.

Jones, B. T., Jones, B. C., Smith, H., \& Copley, N. (2003). A flicker paradigm for inducing change blindness reveals alcohol and cannabis information processing biases in social users. Addiction, 98, 235-244.

JunghöFer, M., Elbert, T., Tucker, D. M., \& Braun, C. (1999). The polar average reference effect: A bias in estimating the head surface integral in EEG recording. Clinical Neurophysiology, 110, 11491155 .

Kimura, M., Katayama, J., \& Murohashi, H. (2005). Positive difference in ERPs reflects independent processing of visual changes. Psychophysiology, 42, 369-379.

Kimura, M., Katayama, J., \& Murohashi, H. (2006a). An ERP study of visual change detection: Effects of magnitude of spatial frequency changes on the change-related posterior positivity. International Journal of Psychophysiology, 62, 14-23.

Kimura, M., Katayama, J., \& Murohashi, H. (2006b). Independent processing of visual stimulus changes in ventral and dorsal stream features indexed by an early positive difference in event-related brain potentials. International Journal of Psychophysiology, 59, 141-150.

Kimura, M., Katayama, J., \& Murohashi, H. (2006c). Probability- independent and -dependent ERPs reflecting visual change detection. Psychophysiology, 43, 180-189.

Korvisto, M., \& Revonsuo, A. (2003). An ERP study of change detection, change blindness, and visual awareness. Psychophysiology, 40, 423-429.

Mazza, V., Turatto, M., \& Sarlo, M. (2005). Rare stimuli or rare changes: What really matters for the brain? NeuroReport, 16, 10611064.

McCarthy, G., \& Wood, C. C. (1985). Scalp distributions of eventrelated potentials: An ambiguity associated with analysis of variance models. Electroencephalography \& Clinical Neurophysiology, 62, 203-208.

Pazo-Alvarez, P., Cadaveira, F., \& Amenedo, E. (2003). MMN in the visual modality: A review. Biological Psychology, 63, 199-236.

Rensink, R. A. (2002). Change detection. Annual Review of Psychology, 53, 245-277.

Rossion, B., Collins, D., Goffaux, V., \& Curran, T. (2007). Longterm expertise with artificial objects increases visual competition with early face categorization processes. Journal of Cognitive Neuroscience, 19, 543-555.

Simons, D. J., \& Rensink, R. A. (2005). Change blindness: Past, present, and future. Trends in Cognitive Sciences, 9, 16-20.

TANaKa, J. W., \& Curran, T. (2001). A neural basis for expert object recognition. Psychological Science, 12, 43-47.

Werner, S., \& Thies, B. (2000). Is "change blindness" attenuated by domain-specific expertise? An expert-novices comparison of change detection in football images. Visual Cognition, 7, 163-173.

Wong, A. C., Gauthier, I., Woroch, B., DeBuse, C., \& Curran, T. (2005). An early electrophysiological response associated with expertise in letter perception. Cognitive, Affective, \& Behavioral Neuroscience, 5, 306-318.

(Manuscript received December 26, 2007; revision accepted for publication September 10,2008.) 